Article

\title{
Nutrient Composition of Demersal, Pelagic, and Mesopelagic Fish Species Sampled Off the Coast of Bangladesh and Their Potential Contribution to Food and Nutrition Security-The EAF-Nansen Programme
}

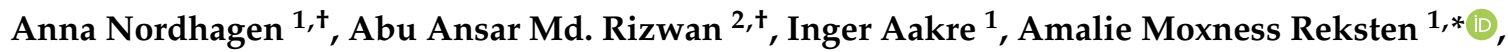 \\ Lauren Michelle Pincus ${ }^{3}$ (D) Annbjørg Bøkevoll ${ }^{1}$, Al Mamun ${ }^{4}$, Shakuntala Haraksingh Thilsted ${ }^{3}$, \\ Thaung Htut ${ }^{5}$, Thiruchenduran Somasundaram ${ }^{6, \ddagger}$ iD and Marian Kjellevold ${ }^{1}$ D \\ 1 Institute of Marine Research, P.O. Box 2029 Nordnes, 5817 Bergen, Norway; \\ nordhagen_94@hotmail.com (A.N.); inger.aakre@hi.no (I.A.); annbjorg.bokevoll@hi.no (A.B.); \\ marian.kjellevold@hi.no (M.K.) \\ 2 Health and Nutrition, Social Assistance and Rehabilitation for the Physically Vulnerable (SARPV), \\ Cox's Bazar 4700, Bangladesh; aamdrizwan@gmail.com \\ 3 WorldFish, Jalan Batu Maung, Batu Maung, Bayan Lepas, Penang 11960, Malaysia; \\ 1.pincus@cgiar.org (L.M.P.); s.thilsted@cgiar.org (S.H.T.) \\ 4 Marine Fisheries Survey Management Unit, Department of Fisheries, CGO Building-2, Agrabad, \\ Chattogram 4100, Bangladesh; mamunbau08@yahoo.com \\ 5 Wildlife Conservation Society-Myanmar Program, P.O. Box Kamayut, Yangon 11041, Myanmar; \\ thtut@wcs.org \\ 6 Institute of Postharvest Technology, National Aquatic Resources Research and Development \\ Agency (NARA), P.O. Box Colombo 01500, Sri Lanka; tsomasundaram@deakin.edu.au \\ * Correspondence: amalie.moxness.reksten@hi.no; Tel.: +47-975-83-269 \\ + These authors contributed equally to this work. \\ $\ddagger$ Current affiliation: P.O. Box 423, Deakin Residence, P-Building, Deakin University, Princess Highway, \\ Warrnambol, Victoria 3280, Australia.
}

Received: 30 April 2020; Accepted: 27 May 2020; Published: 3 June 2020

\begin{abstract}
Fish is a major part of the Bangladeshi diet, but data on the nutrient composition of marine fish species are sparse. Mesopelagic fish may be a new potential resource of food and nutrients; however, nutrient composition data are lacking. The aim of this study was to provide nutrient composition data of fish species sampled off the coast of Bangladesh and determine their potential contribution to recommended nutrient intakes (RNI). Seven species from the pelagic, mesopelagic, and demersal zones were sampled from the coast of Bangladesh with Dr. Fridtjof Nansen in 2018. Three pooled samples containing 15-840 individuals from each species were analysed at the Institute of Marine Research, Norway. The demersal species contained substantially lower concentrations of nearly all nutrients, whereas the mesopelagic species generally were more nutrient dense. All species, except for the demersal species Bombay duck ( $9 \%$ dry matter), were found to contribute $\geq 100 \%$ to the RNI of vitamin $B_{12}$, eicosapentaenoic acid, docosahexaenoic acid, and selenium. All species, except for the demersal fish species, contributed $\geq 25 \%$ to the RNI of six or more nutrients. The data presented in this paper are an important contribution to the Bangladeshi food composition table and contribute to the understanding of fish as an important source of micronutrients.
\end{abstract}

Keywords: fish; nutrient composition; recommended nutrient intakes; micronutrients; food and nutrition security; Bangladesh; Bay of Bengal; marine; food composition data; mesopelagic 


\section{Introduction}

Seafood is considered a high protein food and a major source of fatty acids, such as the very long-chain omega-3 polyunsaturated fatty acids eicosapentaenoic acid (EPA) and docosahexaenoic acid (DHA). Fish is also a rich dietary source of several micronutrients, such as vitamin $A$, vitamin $B_{12}$, vitamin D, calcium, iodine, selenium, and zinc [1-3]. Both freshwater and marine fish are popular among the Bangladeshi people; fish is readily available, well-liked, and less expensive than other animal-source foods in Bangladesh [4,5]. Fish is the third most commonly consumed food in the Bangladeshi diet after rice and vegetables [4] and is by far the most important source of animal protein, accounting for approximately $60 \%$ of total animal protein intake per capita per year [5-8]. In the past two decades, the consumption of fish in Bangladesh has increased because of the rapid expansion of aquaculture $[5,9,10]$. However, malnutrition remains widespread, with a $36 \%$ prevalence rate of stunting and a $14 \%$ prevalence rate of wasting in children under 5 years of age- both of which are among the highest globally [11,12]. Undernutrition is exacerbated by poor dietary diversity and micronutrient deficiencies continue to be a major problem in the country $[5,10,13]$. Vitamin A, vitamin $B_{12}$, zinc, iodine, and folate deficiencies, as well as maternal and child anaemia, are particular public health issues and are highly prevalent [9].

Food composition data (FCD) represent a basic and essential tool for planning interventions to improve health, nutrition, and food and nutrition security in all populations [14]. FCD are used in all aspects of nutrition: food labeling and diet formulation, assessment of nutrient intake and requirements, education and research, estimations of food and nutrition security prevalence, and public health policy formulation [14,15]. The first food composition table (FCT) for Bangladesh was developed in 1977 and consisted of a combination of borrowed and analytical data. The FCT lacked proper documentation of the origin of the data and the analytical methods utilised. It also contained a large number of missing nutrient values and lacked precise descriptions of the foods. In 2013, as a response to the long-standing demands from several institutions in the health, nutrition, education, and agriculture sectors, Bangladesh developed the new and improved FCT in use today. This FCT contains 381 foods and 18 nutrients, with most of the data given for raw foods. The foods are classified into 15 food groups, with "Fish, shellfish, and their products" being a major group consisting of 72 food items. Only nine marine fish species are included in the FCT, whereas around 511 various marine fish species exist in the marine waters of Bangladesh [16]. As stated in the FCT, there is still a major lack of knowledge on the nutrient composition of fish species available for consumption in Bangladesh [17].

Though Bangladesh has huge marine water resources, it is estimated that approximately $15 \%$ of the country's total fish production stems from marine capture fisheries, with aquaculture presently providing more than half of the fish available for direct human consumption $[8,18]$. According to official statistics, the most commonly consumed fish species are all of freshwater origin and includes species such as tilapia, catfish, and mrigal carp. However, these data may fail to include a major part of the total fish production, which includes small indigenous fish species, often thought of as "weed" fish [19,20]. The most commonly consumed marine species are hilsa shad and other shads [21], closely followed by Bombay duck [22]. In the 1970s and 1980s, several surveys examined the status of the marine fish stocks in the country, suggesting that these might be over-exploited, but no recent or comprehensive knowledge is available [18]. To reduce existing undernutrition and micronutrient deficiencies and contribute to food and nutrition security, new marine resources could be explored, not only directly for human consumption, but also indirectly as feed ingredients for use in the flourishing aquaculture sector. Mesopelagic fish, the smallest and most abundant pelagic fish species residing in the mesopelagic zone stretching from 200 to $1000 \mathrm{~m}$ depth during daytime, may represent an important and sustainable source of marine nutrients, however, present research and knowledge on these species are particularly scarce [23-25]. The aim of this study was to quantify the nutritional composition of pelagic, mesopelagic, and demersal fish species from the marine waters of Bangladesh to fill knowledge gaps in the existing FCD. In addition, the potential contribution of these fish species to recommended nutrient intakes as a mean to evaluate their value to food and nutrition security 
in Bangladesh was assessed. The micronutrients considered in relation to the RNI were vitamin A, vitamin $B_{12}$, vitamin $D$, calcium, iron, iodine, selenium, and zinc, and the essential fatty acids EPA and DHA.

\section{Materials and Methods}

This paper uses data collected through the scientific surveys with the research vessel (R/V) Dr. Fridtjof Nansen as part of the collaboration between the EAF-Nansen Programme and the Department of Fisheries (DoF) in Bangladesh. The EAF-Nansen Programme is a partnership between the Food and Agriculture Organization of the United Nations (FAO), the Norwegian Agency for Development Cooperation (Norad), and the Institute of Marine Research (IMR), Norway, for sustainable management of the fisheries of partner countries.

\subsection{Fish Sampling}

Sampling of fish was performed during a survey conducted by R/V Dr. Fridtjof Nansen in the Bay of Bengal off the coast of Bangladesh. Surveying took place over twelve days in August 2018. Sampling of fish was carried out using pelagic (MultiPelt 624) and bottom trawls (Gisund Super bottom trawl). "Pelagic" refers to the pelagic zone of the ocean, and fish in this habitat zone occupy the open water column that is not near the shore nor the bottom, whereas demersal fish live on or near the bottom. The sampling location for each species is presented in a map in Figure 1. For each trawl, the fish were sorted, and the species identified by local taxonomists using the FAO Species Guides and catalogues [26-28]. A list of prioritised fish species based on the most commonly consumed species in Bangladesh was generated at the beginning of the survey by Bangladeshi scientists and taxonomists. Mesopelagic species were included in the study due to the limited knowledge on the nutrient composition of these species, even though they are currently not widely consumed. Samples of pelagic and demersal fish species were prepared according to local dietary habits: as fillets with skin and bones, but without the head, tail, and viscera (Table 1). The mesopelagic species were prepared whole, including the head, skin, tail, and viscera. Three pooled samples, consisting of a minimum of five individual fish in each sample, were prepared for nutrient analyses at the IMR in Bergen, Norway. The samples were homogenised using a food processor (Braun Multiquick 7 K3000, Kronberg im Taunus, Germany) and stored as wet samples at $-20{ }^{\circ} \mathrm{C}$ in the on-board freezer. After a minimum of $12 \mathrm{~h}$ in the freezer, a sub-sample of each wet sample was freeze-dried for $72 \mathrm{~h}\left(24 \mathrm{~h}\right.$ at $-50{ }^{\circ} \mathrm{C}$, immediately followed by $48 \mathrm{~h}$ at $+25^{\circ} \mathrm{C}$, with a vacuum of $0.2-0.01 \mathrm{mbar}$ ), using a Labconco FreeZone 18 litres freeze-dryer (mod. 7750306, Kansas City, MO, USA) and the dry matter (\%) was determined. Freeze-dried samples were then homogenised once more using a knife mill (Retch Grindomix GM 200, Haan, Germany). All samples were packed in plastic bags and stored in insulated boxes in the vessel's freezer $\left(-20^{\circ} \mathrm{C}\right)$ until shipment by air cargo to the IMR laboratories in Bergen, Norway, where the samples were stored at $-80^{\circ} \mathrm{C}$, pending analyses. A detailed description of the sampling procedures can be found in Reksten et al. [29]. 
Table 1. Identification details and overview of fish species sampled from Bangladesh.

\begin{tabular}{|c|c|c|c|c|c|c|}
\hline Common Name & Scientific Name & Local Name & Habitat & Fish Tissue Sampled & $\begin{array}{c}\text { Number of Pooled } \\
\text { Samples }\end{array}$ & $\begin{array}{c}\text { Number of Fish in Each } \\
\text { Pooled Sample }\end{array}$ \\
\hline Fringescale sardinella 1 & Sardinella fimbriata & Chapila & Pelagic & Fillet with skin and bones & 3 & $23^{a}$ \\
\hline Fringescale sardinella $^{2}$ & Sardinella fimbriata & Chapila & Pelagic & Fillet with skin and bones & 3 & 25 \\
\hline Fringescale sardinella ${ }^{3}$ & Sardinella fimbriata & Chapila & Pelagic & Fillet with skin and bones & 3 & 25 \\
\hline Slender rainbow sardine ${ }^{1}$ & Dussumieria elopsoides & Maricha & Pelagic & Fillet with skin and bones & 3 & 25 \\
\hline Slender rainbow sardine ${ }^{2}$ & Dussumieria elopsoides & Maricha & Pelagic & Fillet with skin and bones & 3 & 25 \\
\hline Slender rainbow sardine ${ }^{3}$ & Dussumieria elopsoides & Maricha & Pelagic & Fillet with skin and bones & 3 & $23^{a}$ \\
\hline Torpedo scad & Megalaspis cordyla & Kauwa & Pelagic & Fillet with skin and bones & 3 & 5 \\
\hline Spinycheek lanternfish & Benthosema fibulatum & Puiya & Mesopelagic & Whole fish & 3 & 250 \\
\hline Unicorn cod & Bregmaceros mcclellandi & $-\mathrm{b}$ & Mesopelagic & Whole fish & 3 & 280 \\
\hline Bombay duck $^{1}$ & Harpadon nehereus & Loittya & Demersal & Fillet with skin and bones & 3 & 25 \\
\hline Bombay duck $^{2}$ & Harpadon nehereus & Loittya & Demersal & Fillet with skin and bones & 3 & 20 \\
\hline Longfin mojarra & Pentaprion longimanus & Dom Mach & Demersal & Fillet with skin and bones & 3 & 25 \\
\hline
\end{tabular}

a One pooled sample consisted of 22 fish, whereas the other two consisted of 23 fish. ${ }^{b}$ Local name of fish species not available. ${ }^{1,2,3}$ Same species sampled multiple times from different locations. 


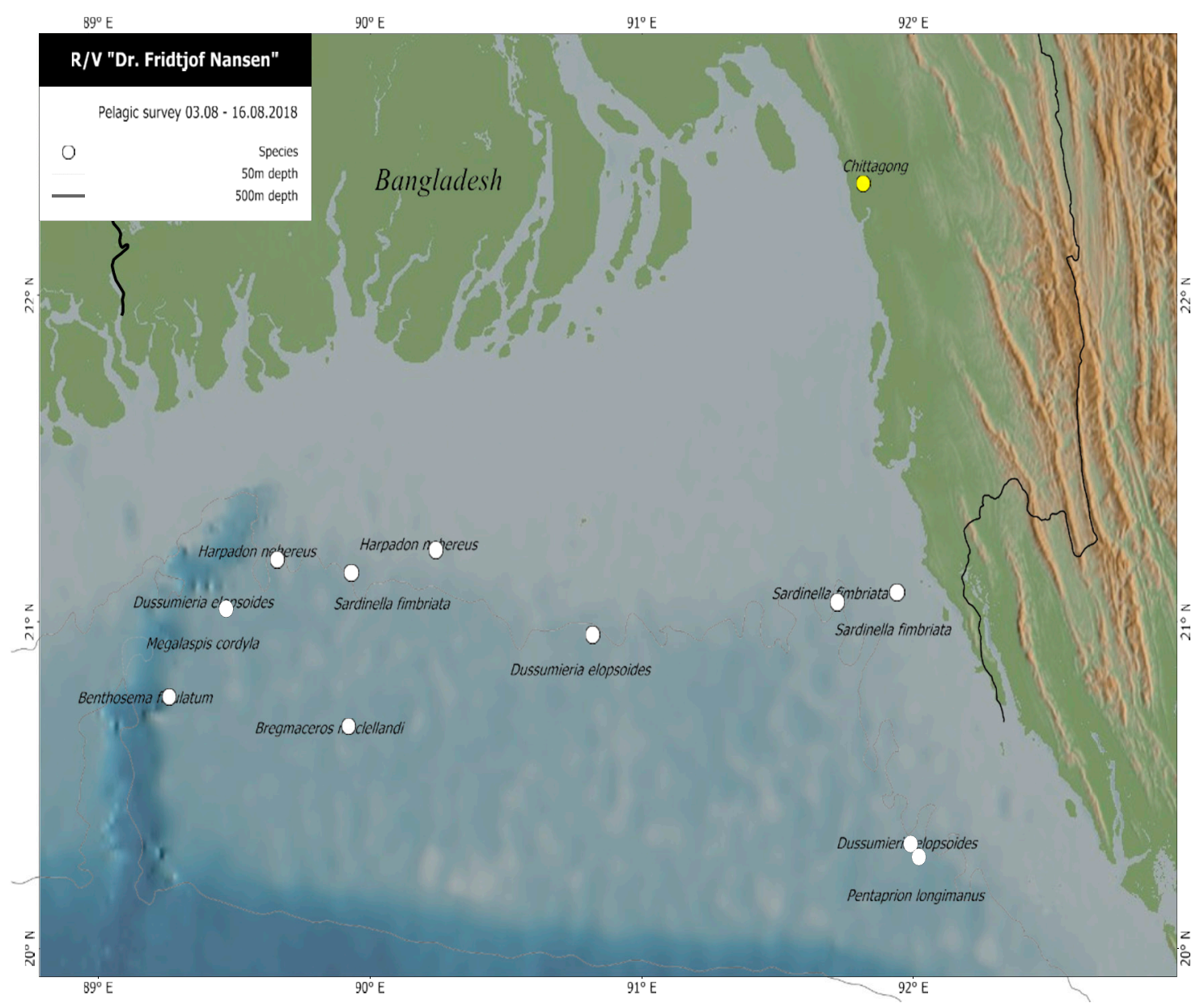

Figure 1. Sampling locations of the included fish species during the 2018 Nansen survey in Bangladesh, the Bay of Bengal.

\subsection{Analytical Methods}

The determination of nutrients in the sampled species was carried out at the IMR laboratories. Details of the analytical methods are described by Reksten et al. [29]. Crude fat was extracted with ethyl acetate and filtered before the solvent was evaporated and the fat residue was weighed. The method is standardised as a Norwegian Standard, NS 9402 [30]. Crude protein was determined by burning the material in pure oxygen gas in a combustion tube at $950{ }^{\circ} \mathrm{C}$. Nitrogen $(\mathrm{N})$ was detected with a thermal conductively detector and the content of nitrogen was calculated from an estimated average of $16 \% \mathrm{~N}$ per $100 \mathrm{~g}$ protein. The following formula was used: $\mathrm{N} \mathrm{g} / 100 \mathrm{~g} \times 6.25=$ g protein $/ 100 \mathrm{~g}$, in accordance with the method accredited by the AOAC Official Methods of Analysis [31]. For analysis of fatty acids, lipids from the samples were extracted according to Folch et al. [32]. After filtering, the remaining samples were saponified and methylated. The fatty acid composition of total lipids was analysed as previously described [33,34]. The samples for the determination of vitamin $\mathrm{A}_{1}$ (sum of all trans-retinol and 13-, 11-, 9 cis retinol) and vitamin $\mathrm{A}_{2}$ (3,4 didehydro-all-trans-retinol) were saponified and the unsaponifiable material was extracted. Vitamin $\mathrm{A}_{1}$ and $\mathrm{A}_{2}$ concentrations were determined by high-performance liquid chromatography (HPLC, normal phase) using a Photo Diode Array (PDA) detector. The content of all-trans-retinol was calculated by external calibration (standard curve) [35], and the content of the other vitamin A forms was calculated based on the external calibration curve for all-trans-retinol multiplied by a correction factor. The samples for the determination of vitamin $\mathrm{D}_{3}$ were saponified and the unsaponifiable material was extracted and then purified on a preparative HPLC column. The fraction containing $\mathrm{D}_{2}$ (ergocalciferol) and $\mathrm{D}_{3}$ (cholecalciferol) was pooled (normal 
phase). This fraction was injected on an analytical HPLC column (reverse phase). Vitamin $\mathrm{D}_{2} / \mathrm{D}_{3}$ was determined by an UV detector. The content of vitamin $\mathrm{D}_{3}$ was calculated using an internal standard (vitamin $\mathrm{D}_{2}$ ) [36]. Vitamin $\mathrm{B}_{12}$ (cobalamin) was released from the sample by extraction (autoclaving in acetate buffer) and mixed with growth medium, added a microorganism (Lactobacillus delbruecki -ATCC 4797), and incubated at $37^{\circ} \mathrm{C}$ for $22 \mathrm{~h}$. The vitamin $\mathrm{B}_{12}$ content was calculated by comparing the growth of the organism in the unknown samples, with the growth of the organism in known standard concentrations with turbidimetric reading (Optical Density, OD, v/575 nm) [37]. The concentrations of minerals (selenium $(\mathrm{Se})$, zinc $(\mathrm{Zn})$, iron $(\mathrm{Fe})$, calcium $(\mathrm{Ca})$, potassium $(\mathrm{K})$, magnesium $(\mathrm{Mg})$, phosphorus $(\mathrm{P})$, and sodium $(\mathrm{Na})$ ) were determined by inductively coupled plasma-mass spectrometry (iCapQ ICP-MS, ThermoFisher Scientific, Waltham, MA, USA) equipped with an auto-sampler (FAST SC-4Q DX, Elemental Scientific, Omaha, NE, USA) after wet digestion in a microwave oven (UltraWave, Milestone, Sorisole, Italy), as described by Julshamn et al. [38]. The concentration of these minerals was quantified using an external standard curve in addition to an internal standard [39]. Three slightly different methods were applied: (1) for $\mathrm{Ca}, \mathrm{Na}, \mathrm{K}, \mathrm{Mg}$, and $\mathrm{P}$, using scandium (Sc) as the internal standard, (2) for $\mathrm{Zn}$ and Se, using rhodium (Rh) as the internal standard, and (3) for iodine (I), tellurium (Te) was used as the internal standard. For the determination of I, the sample preparation was a basic extraction with tetramethylammonium hydroxide (TMAH) before ICP-MS analysis.

\subsection{Data Management and Presentation of Analytical Data}

All analytical values were exported from Laboratory Information Management System (LIMS) into Microsoft ${ }^{\circledR}$ Office Excel 2013 version 15.0 for calculations of means and standard deviations (SD). For species that were sampled from multiple locations, a mean value for all samples of each species was calculated. Single values $<$ Limit of Quantitation (LOQ) are presented as below the respective value for each analyte. For vitamin $A_{2}$, four values were below the LOQ of $0.5 \mu \mathrm{g} / 100 \mathrm{~g}$, whereas for vitamin $\mathrm{D}$, five values were below the LOQ of $1.0 \mu \mathrm{g} / 100 \mathrm{~g}$. When calculating means and SD, values below LOQ were entered into the dataset as the respective LOQ-value divided by two.

\subsection{Calculation of Potential Contribution to Recommended Nutrient Intakes}

The potential contribution of each species to recommended nutrient intakes (RNI) of selected micronutrients was calculated within reference to non-pregnant, non-lactating, healthy females of reproductive age (19-50 years). A $100 \mathrm{~g}$ portion of raw, edible parts of the various fish species was used to estimate the percentage of the RNI for the various micronutrients, using the vitamin and mineral requirements published by WHO and FAO [40]. For the fatty acids EPA and DHA, the Dietary Reference Values from the European Food Safety Authority (EFSA) were used [41]. For species that were sampled at several different locations, the mean of all samples was calculated and used as the reference value for that species. Although variable for different nutrients, the RNI values were calculated assuming a dietary bioavailability of $100 \%$.

\section{Results}

\subsection{Sample Characteristics}

This paper includes a total of five commonly consumed marine fish species and two mesopelagic fish species. For two of the pelagic species, samples from three separate locations were analysed, and for one of the demersal species, samples from two separate locations were analysed. The weight, length, and habitat characteristics of the sampled species are described in Table 2. 
Table 2. Mean physical parameters of fish species sampled from Bangladesh.

\begin{tabular}{|c|c|c|}
\hline Common name & Weight (g) ${ }^{a}$ & Length $(\mathrm{cm})^{a}$ \\
\hline \multicolumn{3}{|l|}{ Pelagic } \\
\hline Fringescale sardinella ${ }^{1}$ & 35.2 & 16.1 \\
\hline Fringescale sardinella $^{2}$ & 40.3 & 16.4 \\
\hline Fringescale sardinella ${ }^{3}$ & 43.5 & 16.4 \\
\hline Slender rainbow sardine ${ }^{1}$ & 49.7 & 17.0 \\
\hline Slender rainbow sardine ${ }^{2}$ & 72.6 & 20.3 \\
\hline Slender rainbow sardine ${ }^{3}$ & 67.8 & 19.3 \\
\hline Torpedo scad & 114.6 & 25.2 \\
\hline \multicolumn{3}{|l|}{ Mesopelagic } \\
\hline Spinycheek lanternfish & 0.6 & $<5$ \\
\hline Unicorn cod & 0.5 & $<6$ \\
\hline \multicolumn{3}{|l|}{ Demersal } \\
\hline Bombay duck ${ }^{1}$ & 110.3 & 25.5 \\
\hline Bombay duck ${ }^{2}$ & 117.7 & 24.2 \\
\hline Longfin mojarra & 20.8 & 11.4 \\
\hline
\end{tabular}

${ }^{a}$ Weight and length measurements are expressed as the mean of one pooled sample consisting of $n$ number of fish. 1,2,3 Same species sampled multiple times from different locations.

\subsection{Pelagic Fish Species}

Table 3 describes the proximate composition of the sampled fish species. The pelagic species fringescale sardinella, slender rainbow sardine, and torpedo scad had the highest protein content of the sampled species (21/100 g raw, edible parts). Furthermore, a sample of fringescale sardinella presented the highest content of zinc compared to the rest of the species $(2.0 \mathrm{mg} / 100 \mathrm{~g}$ raw, edible parts, Table 4$)$, whereas torpedo scad had the highest content of vitamin $B_{12}(1.1 \mu \mathrm{g} / 100 \mathrm{~g}$ raw, edible parts, Table 5).

Table 3. Analytical values of the proximate composition of the fish species sampled from Bangladesh ${ }^{\mathrm{a}}$.

\begin{tabular}{|c|c|c|c|c|}
\hline Sampled Species & & Dry Matter & Protein & Fat \\
\hline Pelagic & $\mathrm{n}^{\mathrm{b}}$ & $\%$ & $\mathrm{~g} / 100 \mathrm{~g}$ & $\mathrm{~g} / 100 \mathrm{~g}$ \\
\hline Fringescale sardinella $^{1}$ & 3 & $23.6 \pm 0.5$ & $21 \pm 0.0$ & $1.6 \pm 0.2$ \\
\hline Fringescale sardinella ${ }^{2}$ & 3 & $25.0 \pm 0.3$ & $21 \pm 0.6$ & $1.8 \pm 0.1$ \\
\hline Fringescale sardinella ${ }^{3}$ & 3 & $25.3 \pm 0.4$ & $21 \pm 0.6$ & $2.4 \pm 0.4$ \\
\hline Mean of all Fringescale sardinella $1,2,3$ & 9 & $24.6 \pm 0.9$ & $21 \pm 0.5$ & $1.9 \pm 0.4$ \\
\hline Slender rainbow sardine ${ }^{1}$ & 3 & $23.4 \pm 2.2$ & $21 \pm 2.1$ & $1.13 \pm 0.1$ \\
\hline Slender rainbow sardine 2 & 3 & $23.0 \pm 0.3$ & $21 \pm 0.6$ & $1.3 \pm 0.3$ \\
\hline Slender rainbow sardine ${ }^{3}$ & 3 & $24.1 \pm 0.4$ & $21 \pm 0.6$ & $1.9 \pm 0.1$ \\
\hline Mean of all Slender rainbow sardine $1,2,3$ & 9 & $23.5 \pm 1.3$ & $21 \pm 1.1$ & $1.4 \pm 0.4$ \\
\hline Torpedo scad & 3 & $24.6 \pm 1.8$ & $21 \pm 1.5$ & $1.3 \pm 0.2$ \\
\hline \multicolumn{5}{|l|}{ Mesopelagic } \\
\hline Spinycheek lanternfish & 3 & $24.2 \pm 0.5$ & $17 \pm 0.1$ & $3.3 \pm 0.1$ \\
\hline Unicorn cod & 3 & $20.9 \pm 1.1$ & $15 \pm 1.1$ & $1.6 \pm 0.2$ \\
\hline \multicolumn{5}{|l|}{ Demersal } \\
\hline Bombay duck ${ }^{1}$ & 3 & $8.12 \pm 0.2$ & $10 \pm 1.8$ & $0.64 \pm 0.0$ \\
\hline Bombay duck ${ }^{2}$ & 3 & $9.45 \pm 0.9$ & $11 \pm 2.4$ & $0.85 \pm 0.1$ \\
\hline Mean of all Bombay duck 1,2 & 6 & $8.79 \pm 0.9$ & $10 \pm 2.0$ & $0.74 \pm 0.1$ \\
\hline Longfin mojarra & 3 & $23.3 \pm 1.0$ & $19 \pm 1.2$ & $2.5 \pm 0.1$ \\
\hline
\end{tabular}

${ }^{a}$ Values are presented as means \pm standard deviations (SD) of the fish species analysed in triplicates, expressed as the nutrient content per $100 \mathrm{~g}$ raw, edible part. ${ }^{\mathrm{b}}$ Number of pooled samples analysed. Each pooled sample consisted of a minimum of 5 fish. ${ }^{1,2,3}$ Same species sampled multiple times from different locations. 
Table 4. Analytical values of the content of minerals and trace elements in the species sampled from Bangladesh ${ }^{\text {a }}$

\begin{tabular}{|c|c|c|c|c|c|c|c|c|c|c|}
\hline \multirow{2}{*}{ Sampled Species } & \multicolumn{2}{|r|}{$\mathrm{Ca}$} & \multirow{2}{*}{$\begin{array}{l}\mathrm{Na} \\
\mathrm{mg} / 100 \mathrm{~g}\end{array}$} & \multirow{2}{*}{$\begin{array}{l}\mathrm{K} \\
\mathrm{mg} / 100 \mathrm{~g} \\
\end{array}$} & \multirow{2}{*}{$\begin{array}{l}\mathrm{Mg} \\
\mathrm{mg} / 100 \mathrm{~g}\end{array}$} & \multirow{2}{*}{$\begin{array}{l}P \\
\mathrm{mg} / 100 \mathrm{~g}\end{array}$} & \multirow{2}{*}{$\begin{array}{l}\mathrm{I} \\
\mu \mathrm{g} / 100 \mathrm{~g}\end{array}$} & \multirow{2}{*}{$\begin{array}{l}\text { Se } \\
\mu \mathrm{g} / 100 \mathrm{~g}\end{array}$} & \multirow{2}{*}{$\begin{array}{l}\mathrm{Zn} \\
\mathrm{mg} / 100 \mathrm{~g}\end{array}$} & \multirow{2}{*}{$\begin{array}{l}\mathrm{Fe} \\
\mathrm{mg} / 100 \mathrm{~g}\end{array}$} \\
\hline & $n^{b}$ & $\mathrm{mg} / \mathbf{1 0 0} \mathrm{g}$ & & & & & & & & \\
\hline \multicolumn{11}{|l|}{ Pelagic } \\
\hline Fringescale sardinella ${ }^{1}$ & 3 & $597 \pm 6$ & $80 \pm 2$ & $470 \pm 10$ & $43 \pm 1$ & $583 \pm 15$ & $23.7 \pm 0.6$ & $113.3 \pm 5.8$ & $1.9 \pm 0.2$ & $2.2 \pm 0.1$ \\
\hline Fringescale sardinella ${ }^{2}$ & 3 & $473 \pm 117$ & $84 \pm 1$ & $500 \pm 10$ & $44 \pm 2$ & $543 \pm 51$ & $66.7 \pm 15.3$ & $116.7 \pm 5.8$ & $2.0 \pm 0.1$ & $2.4 \pm 0.2$ \\
\hline Fringescale sardinella ${ }^{3}$ & 3 & $527 \pm 244$ & $73 \pm 2$ & $487 \pm 12$ & $43 \pm 3$ & $557 \pm 117$ & $25.3 \pm 0.6$ & $99 \pm 9.3$ & $1.9 \pm 0.2$ & $1.9 \pm 0.2$ \\
\hline $\begin{array}{l}\text { Mean of all Fringescale } \\
\text { sardinella } 1,2.3\end{array}$ & 9 & $532 \pm 146$ & $79 \pm 5$ & $486 \pm 16$ & $43 \pm 2$ & $561 \pm 67$ & $38.6 \pm 22.4$ & $110 \pm 10$ & $1.9 \pm 0.2$ & $2.2 \pm 0.3$ \\
\hline Slender rainbow sardine ${ }^{1}$ & 3 & $353 \pm 90$ & $76 \pm 7$ & $503 \pm 49$ & $46 \pm 4$ & $473 \pm 67$ & $16.3 \pm 0.6$ & $84 \pm 11$ & $1.5 \pm 0.2$ & $1.1 \pm 0.2$ \\
\hline Slender rainbow sardine ${ }^{2}$ & 3 & $280 \pm 56$ & $62 \pm 3$ & $500 \pm 20$ & $43 \pm 2$ & $430 \pm 26$ & $11.0 \pm 0.0$ & $70 \pm 3.8$ & $1.4 \pm 0.1$ & $1.0 \pm 0.1$ \\
\hline Slender rainbow sardine ${ }^{3}$ & 3 & $277 \pm 31$ & $83 \pm 6$ & $513 \pm 15$ & $46 \pm 1$ & $443 \pm 15$ & $16.7 \pm 0.6$ & $75 \pm 2.0$ & $1.5 \pm 0.1$ & $0.9 \pm 0.0$ \\
\hline $\begin{array}{l}\text { Mean of all Slender rainbow } \\
\text { sardine } \mathbf{1}^{1,2,3}\end{array}$ & 9 & $303 \pm 67$ & $74 \pm 10$ & $506 \pm 28$ & $45 \pm 3$ & $449 \pm 41$ & $14.7 \pm 2.8$ & $76 \pm 8.4$ & $1.5 \pm 0.1$ & $1.0 \pm 0.1$ \\
\hline $\begin{array}{l}\text { Torpedo scad } \\
\text { Mesopelagic }\end{array}$ & 3 & $670 \pm 549$ & $70 \pm 17$ & $417 \pm 21$ & $37 \pm 8$ & $580 \pm 282$ & $41.7 \pm 16.3$ & $63 \pm 0.0$ & $0.1 \pm 0.3$ & $2.4 \pm 0.1$ \\
\hline Spinycheek lanternfish & 3 & $940 \pm 69$ & $230 \pm 0$ & $303 \pm 6$ & $60 \pm 1$ & $577 \pm 12$ & $160.0 \pm 26.5$ & $120.0 \pm 0.0$ & $1.5 \pm 0.1$ & $2.5 \pm 0.1$ \\
\hline $\begin{array}{l}\text { Unicorn cod } \\
\text { Demersal }\end{array}$ & 3 & $1033 \pm 58$ & $260 \pm 10$ & $327 \pm 15$ & $57 \pm 2$ & $613 \pm 12$ & $160.0 \pm 20.0$ & $67 \pm 3.5$ & $1.1 \pm 0.0$ & $4.9 \pm 5.3$ \\
\hline Bombay duck ${ }^{1}$ & 3 & $126 \pm 139$ & $150 \pm 10$ & $177 \pm 21$ & $18 \pm 2$ & $143 \pm 59$ & $9.1 \pm 0.6$ & $15 \pm 1.5$ & $0.3 \pm 0.1$ & $0.2 \pm 0.1$ \\
\hline Bombay duck ${ }^{2}$ & 3 & $46 \pm 45$ & $297 \pm 50$ & $210 \pm 26$ & $20 \pm 1$ & $126 \pm 31$ & $6.7 \pm 0.7$ & $18 \pm 4.0$ & $0.3 \pm 0.0$ & $0.2 \pm 0.0$ \\
\hline Mean of all Bombay duck ${ }^{1,2}$ & 6 & $86 \pm 102$ & $173 \pm 41$ & $193 \pm 28$ & $19 \pm 2$ & $135 \pm 43$ & $7.9 \pm 1.4$ & $17 \pm 3.0$ & $0.3 \pm 0.1$ & $0.2 \pm 0.1$ \\
\hline Longfin mojarra & 3 & $407 \pm 93$ & $78 \pm 6$ & $410 \pm 30$ & $38 \pm 1$ & $437 \pm 32$ & $19.0 \pm 4.4$ & $44 \pm 2.0$ & $0.9 \pm 0.1$ & $0.6 \pm 0.0$ \\
\hline
\end{tabular}

${ }^{a}$ Values are presented as means \pm SD of the fish species analysed in triplicates, expressed as the nutrient content per $100 \mathrm{~g}$ raw, edible part. ${ }^{\mathrm{b}}$ Number of pooled samples analysed. Each pooled sample consisted of a minimum of 5 fish. ${ }^{1,2,3}$ Same species sampled multiple times from different locations. Abbreviations: Ca: calcium, Fe: iron, I: iodine, K: potassium, Mg: magnesium, Na: sodium, P: phosphorus, SD: standard deviation, Se: selenium, Zn: zinc. 
Table 5. Analytical values of the vitamin $A\left(A_{1}+A_{2}\right)$, vitamin $B_{12}$, and vitamin $D$ content in species sampled from Bangladesh ${ }^{\text {a }}$.

\begin{tabular}{|c|c|c|c|c|c|}
\hline \multirow{2}{*}{ Species $^{\text {a }}$} & \multicolumn{2}{|r|}{ Vitamin $\mathbf{A}_{1}$} & \multirow{2}{*}{$\begin{array}{l}\text { Vitamin } A_{2} \\
\mu \mathrm{g} / 100 \mathrm{~g}\end{array}$} & \multirow{2}{*}{$\frac{\text { Vitamin } B_{12}}{\mu \mathrm{g} / \mathbf{1 0 0} \mathrm{g}}$} & \multirow{2}{*}{$\frac{\text { Vitamin D }}{\mu \mathrm{g} / 100 \mathrm{~g}}$} \\
\hline & $n^{b}$ & $\mu \mathrm{g} / 100 \mathrm{~g}$ & & & \\
\hline \multicolumn{6}{|l|}{ Pelagic } \\
\hline Fringescale sardinella ${ }^{1}$ & 3 & $10.3 \pm 0.6$ & $5.6 \pm 2.1$ & $4.1 \pm 0.3$ & $4.7 \pm 0.6$ \\
\hline Fringescale sardinella ${ }^{2}$ & 3 & $12.7 \pm 4.7$ & $8.7 \pm 1.5$ & $11 \pm 3.3$ & $4.0 \pm 1.0$ \\
\hline Fringescale sardinella ${ }^{3}$ & 3 & $14.3 \pm 1.5$ & $11.0 \pm 2.0$ & $14 \pm 2.1$ & $2.7 \pm 0.6$ \\
\hline $\begin{array}{l}\text { Mean of all Fringescale } \\
\text { sardinella } 1,2,3\end{array}$ & 9 & $12.4 \pm 3.0$ & $8.4 \pm 2.9$ & $9.9 \pm 4.9$ & $3.8 \pm 1.1$ \\
\hline Slender rainbow sardine ${ }^{1}$ & 3 & $7.3 \pm 1.5$ & $1.8 \pm 0.2$ & $8.3 \pm 0.4$ & $3.3 \pm 0.6$ \\
\hline Slender rainbow sardine ${ }^{2}$ & 3 & $4.1 \pm 2.4$ & $1.3 \pm 0.5$ & $7.1 \pm 1.4$ & $2.7 \pm 1.2$ \\
\hline Slender rainbow sardine ${ }^{3}$ & 3 & $16.3 \pm 1.5$ & $1.4 \pm 0.3$ & $8.8 \pm 0.5$ & $4.7 \pm 2.1$ \\
\hline $\begin{array}{l}\text { Mean of all Slender rainbow } \\
\text { sardine } 1,2,3\end{array}$ & 9 & $9.3 \pm 5.7$ & $1.5 \pm 0.4$ & $8.1 \pm 1.1$ & $3.6 \pm 1.5$ \\
\hline $\begin{array}{l}\text { Torpedo scad } \\
\text { Mesopelagic }\end{array}$ & 3 & $11.7 \pm 4.0$ & $0.7 \pm 0.2$ & $15 \pm 2.1$ & $2.3 \pm 0.6$ \\
\hline Spinycheek lanternfish & 3 & $133 \pm 49.3$ & $6.2 \pm 2.4$ & $13 \pm 1.5$ & $<1.0^{\mathrm{d}}$ \\
\hline Unicorn cod & 3 & $280 \pm 26.5$ & $8.7 \pm 1.2$ & $7.9 \pm 0.7$ & $2.3 \pm 0.6$ \\
\hline \multicolumn{6}{|l|}{ Demersal } \\
\hline Bombay duck $^{1}$ & 3 & $5.2 \pm 0.8$ & $<0.5^{\mathrm{d}}$ & $1.2 \pm 0.1$ & $<1.0^{\mathrm{d}}$ \\
\hline Bombay duck ${ }^{2}$ & 3 & $9.3 \pm 2.9$ & $<0.5^{\mathrm{d}}$ & $1.7 \pm 0.6$ & $<1.0^{\mathrm{d}}$ \\
\hline Mean of all Bombay duck ${ }^{1,2}$ & 6 & $7.3 \pm 3.0$ & $<0.5^{\mathrm{d}}$ & $1.5 \pm 0.5$ & $<1.0^{\mathrm{d}}$ \\
\hline Longfin mojarra & 3 & $7.3 \pm 2.6$ & $<0.5^{\mathrm{d}}$ & $1.1 \pm 0.2$ & $<1.0^{\mathrm{d}}$ \\
\hline
\end{tabular}

${ }^{a}$ Values are presented as means \pm SD of the 19 fish species analysed in triplicates, expressed as the nutrient content per $100 \mathrm{~g}$ raw, edible part. ${ }^{\mathrm{b}}$ Number of pooled samples analysed. Each pooled sample consisted of a minimum of 5 fish. ${ }^{d}$ Value below LOQ (Vitamin A: $0.5 \mu \mathrm{g} / 100 \mathrm{~g}$ raw, edible parts; Vitamin D: $1.0 \mu \mathrm{g} / 100 \mathrm{~g}$ raw, edible parts). $1,2,3$ Same species sampled multiple times from different locations.

\subsection{Mesopelagic Fish Species}

The two mesopelagic species sampled whole, spinycheek lanternfish and unicorn cod, presented higher values of calcium, iodine, selenium, and iron than the rest of the sampled species. The vitamin $\mathrm{A}_{1}$ content was also substantially higher in both mesopelagic species, with a maximum value of $280 \mu \mathrm{g} / 100 \mathrm{~g}$ raw, edible parts in unicorn cod, compared to the pelagic and demersal species. The content of selected fatty acids is presented in Table 6 . Spinycheek lanternfish was the most significant source of DHA and had the second highest content of EPA.

\subsection{Demersal Fish Species}

The content of dry matter, total protein, and fat was considerably lower in Bombay duck compared to the other sampled species. Bombay duck also contained the lowest content of all fatty acids and all minerals, with the exception of zinc. The two demersal species, Bombay duck and longfin mojarra, presented the lowest overall values for all vitamins compared to the rest of the species. For vitamin $\mathrm{A}_{2}$ and vitamin $\mathrm{D}$, all values were $<\mathrm{LOQ}(<0.5 \mu \mathrm{g} / 100 \mathrm{~g}$ raw, edible parts and $<1.0 \mu \mathrm{g} / 100 \mathrm{~g}$ raw, edible parts, respectively). 
Table 6. Analytical values of the fatty acid composition of fish species sampled from Bangladesh a .

\begin{tabular}{|c|c|c|c|c|c|c|c|c|}
\hline \multirow{2}{*}{ Sampled Species } & \multicolumn{2}{|r|}{ Sum SFA } & \multirow{2}{*}{$\begin{array}{l}\text { Sum MUFA } \\
\mathrm{g} / 100 \mathrm{~g}\left(\%^{\mathrm{c}}\right)\end{array}$} & \multirow{2}{*}{$\begin{array}{l}\text { Sum PUFA } \\
\mathrm{g} / 100 \mathrm{~g}\left(\%^{\mathrm{c}}\right)\end{array}$} & \multirow{2}{*}{$\begin{array}{l}\text { Sum n-3 } \\
\mathrm{g} / 100 \mathrm{~g}\left(\%^{\mathrm{c}}\right)\end{array}$} & \multirow{2}{*}{$\begin{array}{l}\text { Sum } n-6 \\
g / 100 \mathrm{~g}\left(\%^{c}\right)\end{array}$} & \multirow{2}{*}{$\begin{array}{l}\text { EPA } \\
g / 100 \mathrm{~g}\left(\%^{c}\right)\end{array}$} & \multirow{2}{*}{$\begin{array}{l}\text { DHA } \\
\mathrm{g} / 100 \mathrm{~g}\left(\%^{\mathrm{c}}\right)\end{array}$} \\
\hline & $n^{b}$ & $\mathrm{~g} / 100 \mathrm{~g}\left(\%^{\mathrm{c}}\right)$ & & & & & & \\
\hline \multicolumn{9}{|l|}{ Pelagic } \\
\hline Fringescale sardinella ${ }^{1}$ & 3 & $0.44 \pm 0.06(39)$ & $0.16 \pm 0.06(14)$ & $0.47 \pm 0.05(42)$ & $0.37 \pm 0.03(34)$ & $0.09 \pm 0.02(7.3)$ & $0.07 \pm 0.01(5.8)$ & $0.27 \pm 0.01(24.2)$ \\
\hline Fringescale sardinella ${ }^{2}$ & 3 & $0.49 \pm 0.03(42)$ & $0.18 \pm 0.03(15)$ & $0.46 \pm 0.02(39)$ & $0.36 \pm 0.01(31)$ & $0.08 \pm 0.01(7.3)$ & $0.08 \pm 0.01(6.7)$ & $0.25 \pm 0.03(21)$ \\
\hline Fringescale sardinella ${ }^{3}$ & 3 & $0.88 \pm 0.14(40)$ & $0.39 \pm 0.10(18)$ & $0.80 \pm 0.10(37)$ & $0.64 \pm 0.08(29)$ & $0.14 \pm 0.01(6.3)$ & $0.20 \pm 0.05(8.9)$ & $0.34 \pm 0.02(16)$ \\
\hline Mean of all Fringescale sardinella $1,2,3$ & 9 & $0.60 \pm 0.23(40)$ & $0.24 \pm 0.13(16)$ & $0.57 \pm 0.18(39)$ & $0.46 \pm 0.14(32)$ & $0.10 \pm 0.03(7.1)$ & $0.11 \pm 0.07(7.1)$ & $0.29 \pm 0.05(20)$ \\
\hline Slender rainbow sardine ${ }^{1}$ & 3 & $0.37 \pm 0.02(40)$ & $0.11 \pm 0.00(12)$ & $0.44 \pm 0.03(46)$ & $0.37 \pm 0.02(39)$ & $0.07 \pm 0.00(7.4)$ & $0.06 \pm 0.00(6.5)$ & $0.28 \pm 0.02(30)$ \\
\hline Slender rainbow sardine ${ }^{2}$ & 3 & $0.36 \pm 0.03(40)$ & $0.11 \pm 0.01(12)$ & $0.40 \pm 0.04(46)$ & $0.34 \pm 0.03(39)$ & $0.06 \pm 0.01(7.0)$ & $0.06 \pm 0.01(6.7)$ & $0.26 \pm 0.02(29)$ \\
\hline Slender rainbow sardine ${ }^{3}$ & 3 & $0.57 \pm 0.03(40)$ & $0.17 \pm 0.01(12)$ & $0.62 \pm 0.06(44)$ & $0.52 \pm 0.05(37)$ & $0.10 \pm 0.01(7.0)$ & $0.10 \pm 0.01(7.2)$ & $0.38 \pm 0.04(27)$ \\
\hline Mean of all Slender rainbow sardine $1,2,3$ & 9 & $0.43 \pm 0.10(40)$ & $0.13 \pm 0.03(12)$ & $0.49 \pm 0.11(45)$ & $0.41 \pm 0.09(38)$ & $0.08 \pm 0.02(7.1)$ & $0.07 \pm 0.02(6.8)$ & $0.31 \pm 0.06(29)$ \\
\hline Torpedo scad & 3 & $0.35 \pm 0.05(34)$ & $0.14 \pm 0.02(14)$ & $0.48 \pm 0.03(47)$ & $0.38 \pm 0.02(37)$ & $0.10 \pm 0.01(10)$ & $0.05 \pm 0.00(5.1)$ & $0.28 \pm 0.01(27)$ \\
\hline \multicolumn{9}{|l|}{ Mesopelagic } \\
\hline Spinycheek lanternfish & 3 & $0.88 \pm 0.00(40)$ & $0.41 \pm 0.00(19)$ & $0.85 \pm 0.01(39)$ & $0.68 \pm 0.01(31)$ & $0.17 \pm 0.00(7.8)$ & $0.16 \pm 0.00(7.2)$ & $0.43 \pm 0.01(19)$ \\
\hline Unicorn cod & 3 & $0.31 \pm 0.02(31)$ & $0.16 \pm 0.01(16)$ & $0.45 \pm 0.02(45)$ & $0.37 \pm 0.02(37)$ & $0.08 \pm 0.00(7.8)$ & $0.06 \pm 0.00(6.1)$ & $0.28 \pm 0.01(28)$ \\
\hline \multicolumn{9}{|l|}{ Demersal } \\
\hline Bombay duck ${ }^{1}$ & 3 & $0.11 \pm 0.02(39)$ & $0.06 \pm 0.01(19)$ & $0.10 \pm 0.01(36)$ & $0.074 \pm 0.01(26)$ & $0.03 \pm 0.00(9.3)$ & $0.02 \pm 0.00(5.8)$ & $0.05 \pm 0.00(17)$ \\
\hline Bombay duck ${ }^{2}$ & 3 & $0.21 \pm 0.07(43)$ & $0.11 \pm 0.04$ & $0.13 \pm 0.01(29)$ & $0.10 \pm 0.01(22)$ & $0.03 \pm 0.00(7.1)$ & $0.03 \pm 0.01(5.6)$ & $0.06 \pm 0.01(14)$ \\
\hline Mean of all Bombay duck ${ }^{1,2}$ & 6 & $0.16 \pm 0.07(41)$ & $0.08 \pm 0.04(21)$ & $0.12 \pm 0.02(32)$ & $0.09 \pm 0.02(24)$ & $0.03 \pm 0.01(8.2)$ & $0.02 \pm 0.01(5.7)$ & $0.06 \pm 0.01(16)$ \\
\hline Longfin mojarra & 3 & $0.74 \pm 0.05(38)$ & $0.45 \pm 0.4(23)$ & $0.67 \pm 0.03(34)$ & $0.52 \pm 0.02(27)$ & $0.15 \pm 0.00(7.6)$ & $0.08 \pm 0.00(4.2)$ & $0.38 \pm 0.02(20)$ \\
\hline
\end{tabular}

${ }^{\mathrm{a}}$ Values are presented as means \pm SD of the fish species analysed in triplicates, expressed as the nutrient content per $100 \mathrm{~g}$ raw, edible parts. ${ }^{\mathrm{b}}$ Number of pooled samples analysed. Each

pooled sample consisted of a minimum of 5 fish. ${ }^{c}$ Values are given in percent of total fatty acids. ${ }^{1,2,3}$ Same species sampled multiple times from different locations. Abbreviations: DHA: docosahexaenoic acid; EPA: eicosapentaenoic acid; MUFA: monounsaturated fatty acids; PUFA: polyunsaturated fatty acids; SD: standard deviation, SFA: saturated fatty acids. 


\subsection{Potential Contribution to Recommended Nutrient Intakes}

The various species' potential contributions to the RNI of women of reproductive age for several micronutrients are presented in Figures 2 and 3. With a consumption portion of $100 \mathrm{~g}$ raw edible parts, all species, except for the two demersal species Bombay duck and longfin mojarra, have the potential to contribute $\geq 100 \%$ to the RNI of vitamin $B_{12}$ for women of reproductive age. Similarly, for EPA and DHA, all species, except for Bombay duck, may contribute $\geq 100 \%$ to the RNI. For vitamin A, most species may only contribute $\leq 10 \%$ to the RNI, except for the two mesopelagic species spinycheek lanternfish and unicorn cod, which were identified to potentially contribute $\geq 25 \%$ and $\geq 50 \%$, respectively. For vitamin $\mathrm{D}$, all sampled species may potentially contribute $\geq 40 \%$ to the RNI, except for the two demersal species, which both presented values $<$ LOQ. Furthermore, all species, except for Bombay duck, may contribute $\geq 100 \%$ to the RNI of selenium. The two mesopelagic species were both identified to contribute $\geq 100 \%$ to the RNI of iodine, whereas the remaining species may contribute $<25 \%$ to the RNI of this mineral. Both mesopelagic species may also contribute $\geq 100 \%$ to the RNI of calcium, whereas the rest of the species were all $<30 \%$ in calcium levels. Neither of the species were identified to have the potential to contribute $>15 \%$ to the RNI of iron.

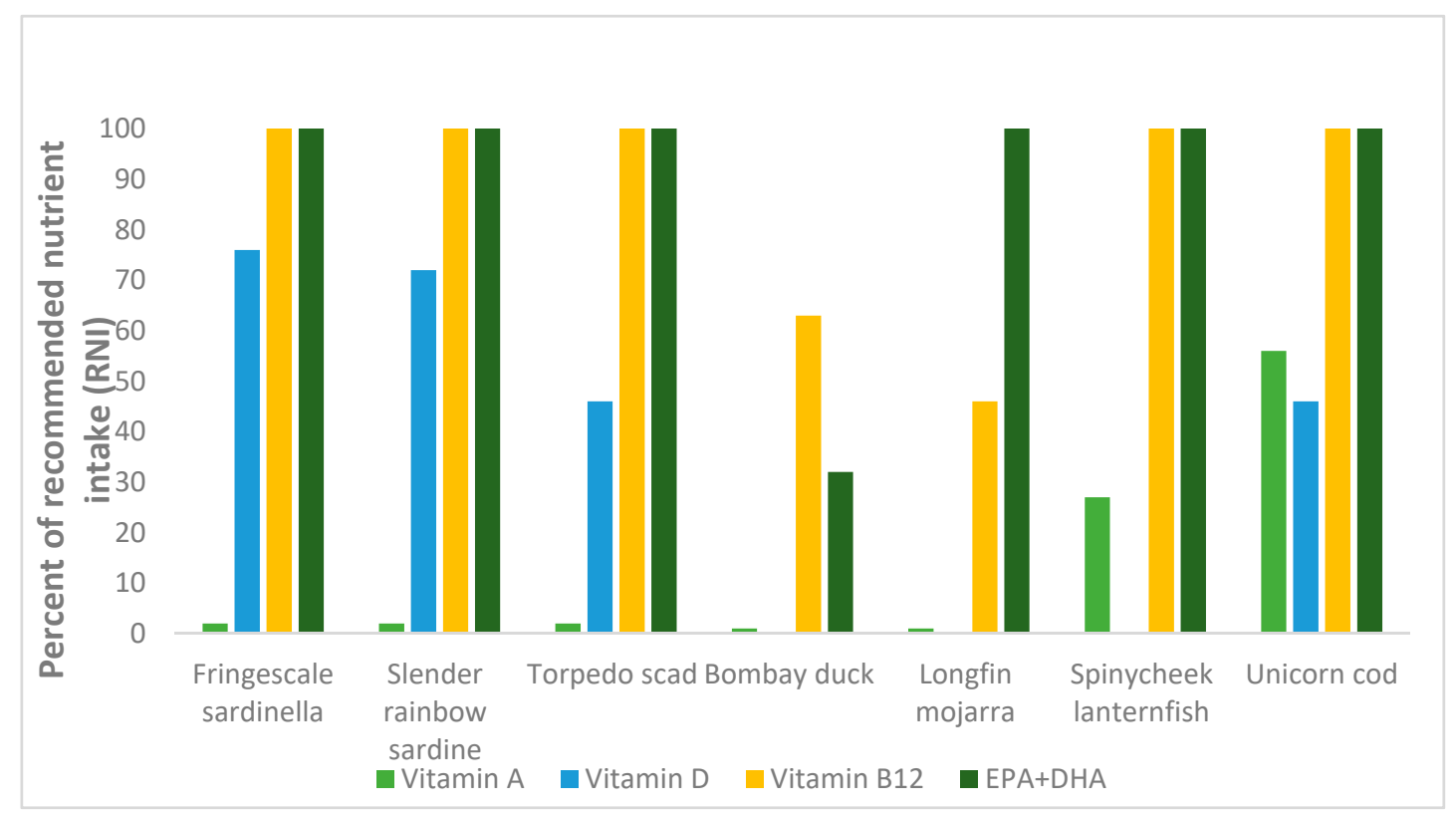

Figure 2. Percentage of recommended nutrient intake (RNI) for women of EPA + DHA and selected vitamins of $100 \mathrm{~g}$ raw, edible parts of Fringescale sardinella (fillet), Slender rainbow sardine (fillet), Torpedo scad (fillet), Bombay duck (fillet), Longfin mojarra (fillet), Spinycheek lanternfish (whole fish), and Unicorn cod (whole fish). 


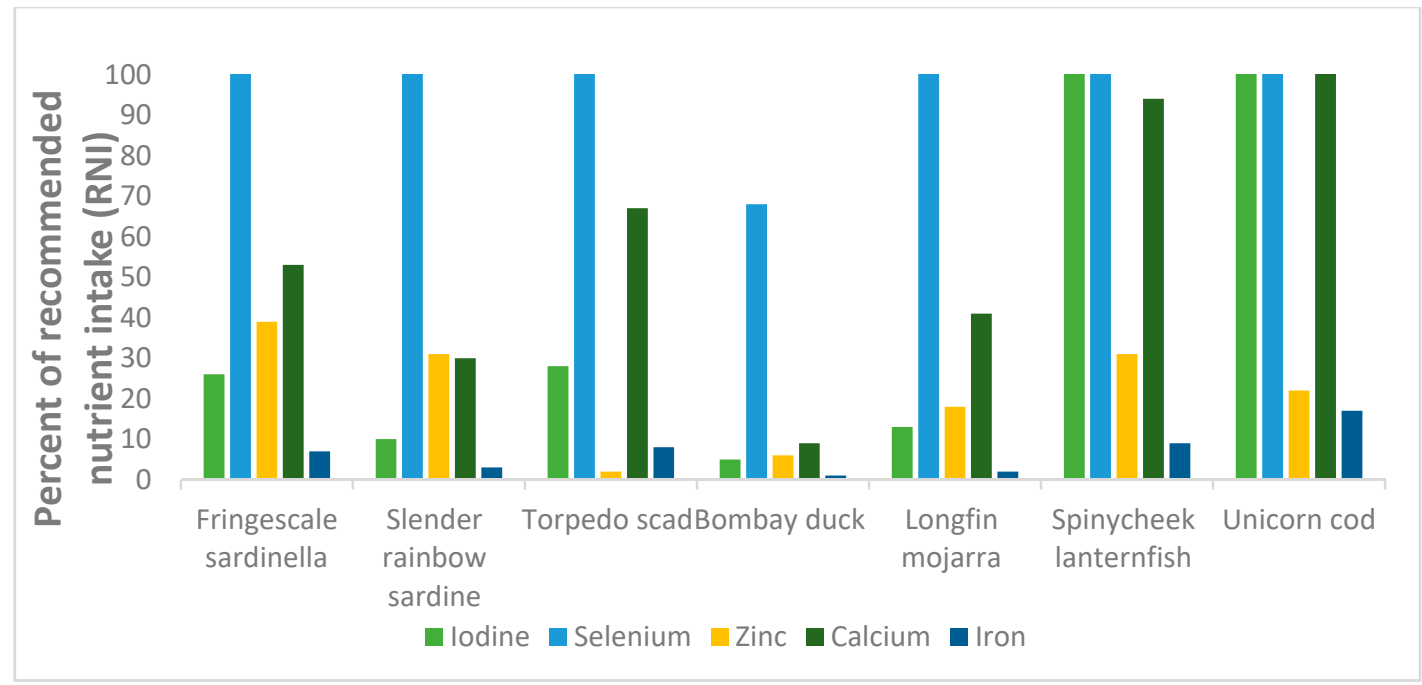

Figure 3. Percentage of recommended nutrient intake (RNI) for women of selected minerals and trace element for $100 \mathrm{~g}$ raw, edible parts of Fringescale sardinella (fillet), Slender rainbow sardine (fillet), Torpedo scad (fillet), Bombay duck (fillet), Longfin mojarra (fillet), Spinycheek lanternfish (whole fish), and Unicorn cod (whole fish).

\section{Discussion}

This paper presents extensive analytical information on the nutrient composition of several marine fish species from the pelagic, mesopelagic, and demersal zones sampled off the coast of Bangladesh in the Bay of Bengal. Of the seven fish species sampled, five are readily available and commonly consumed pelagic and demersal species, whereas the two mesopelagic species represent a group of fish species which have yet to be evaluated for their potential impact on food and nutrition security. Nutrient content varied considerably between the species; with a clear pattern of the mesopelagic species containing the highest concentrations of all micronutrients and the demersal species, particularly Bombay duck, containing substantially lower concentrations of nearly all nutrients. However, it is important to consider that the analyses were performed on raw samples and the water content in this species was particularly high. Several species, particularly the mesopelagic species, were also identified to have the potential to contribute substantially to the RNI of women of reproductive age for micronutrients important for food and nutrition security in Bangladesh. A multitude of studies have previously been conducted to identify the nutrient composition of freshwater fish species, both non-farmed and farmed, in Bangladesh [4,42-49], however, very few studies have been conducted to quantify the nutrients in wild, marine fish species. This study is the first to include comprehensive analyses of the nutrient composition, including the vitamin and mineral composition, of the two mesopelagic species, spinycheek lanternfish and unicorn cod.

\subsection{Pelagic Fish Species}

None of the sampled pelagic species in this paper are included in the Bangladeshi FCT [17]. Torpedo scad is, however, listed in the Malaysian FCT, with nutrient values in agreement with our results for protein, fat, and iron (20.4/100 g, 1.3/100 g, and $2.5 \mathrm{mg} / 100 \mathrm{~g}$, respectively) [50]. However, the values for vitamin $A_{1}$ and calcium are considerably lower $(29 \mu \mathrm{g} / 100 \mathrm{~g}$ and $64.0 \mathrm{mg} / 100 \mathrm{~g}$, respectively) in the Malaysian FCT compared to the values presented in this paper $(11.7 \mu \mathrm{g} / 100 \mathrm{~g}$ and $670 \mathrm{mg} / 100 \mathrm{~g}$ raw, edible parts, respectively). This is probably due to the inclusion and exclusion of various anatomical fish parts in the analyses; only the fillet was included in the samples in the Malaysian FCT [51], whereas we included the fillet with skin and bones, as this is the way these fish species are commonly consumed by the Bangladeshi population. The presence of vitamin $\mathrm{A}_{2}$ in freshwater fish, particularly in the eyes and liver, is well known, however, not many studies have 
evaluated concentrations in marine fish species. For fringescale sardinella, vitamin $\mathrm{A}_{2}$ comprised $54-77 \%$ of the total vitamin A content, thus highlighting the importance of analysing both isomers of the vitamin, even for marine species. The high percentage of vitamin $\mathrm{A}_{2}$ reported in this paper is also in line with the values reported for small indigenous species from Bangladesh [52], but further studies are required to evaluate the occurrence and significance of vitamin $A_{2}$ in marine species. Nearly all calcium and most of the phosphorus and zinc are found primarily in the skin and bones of fish [53-55], thus, the inclusion of these edible parts in the analyses increases the concentration of these micronutrients. This was also demonstrated in a study with Indian fish species, including fringescale sardinella. The content of iron reported was $2.25 \mathrm{mg} / 100 \mathrm{~g}$, which aligns with the values presented in this paper ( $2.2 \mathrm{mg} / 100 \mathrm{~g}$ raw, edible parts), whereas the calcium content of $164.3 \mathrm{mg} / 100 \mathrm{~g}$ reported in their study is substantially lower than the results presented in this paper $(532 \mathrm{mg} / 100 \mathrm{~g}$ raw, edible parts). This difference in calcium content is likely also due to the difference in which anatomical parts of the fish were included in the analyses; they included only the fillet, thus excluding the calcium-rich bones and skin of the fish [56]. The calcium in small fish species commonly consumed whole have been confirmed to be of high bioavailability [53], with a similar absorption rate to that of milk [57].

\subsection{Mesopelagic Fish Species}

Mesopelagic species are small (usually $2-15 \mathrm{~cm}$ in length), deteriorate quickly after harvest and are presently not considered directly suitable for human consumption due to their unknown content of undesirable substances such as wax esters [23,24]. However, it should be recognised that, if unsuitable for direct human consumption, mesopelagic species may still be acceptable as ingredients in fish feed [23]. With the expanding aquaculture industry comes the need for wild-harvesting of marine fatty acids-a demand that mesopelagic species have been suggested to supply [58]. Myctophids, or lanternfish (one of the species also included in this paper), are the most abundant group of mesopelagic fish in the world's oceans, and have, in a study by El-Mowafi et al., previously been identified with a satisfactory level of nutrients and a low level of contaminants and wax esters, and are thus presumed to be qualified to be utilised as ingredients in fish feed [59]. Lanternfish have also in previous studies been recognised as a highly attractive source of raw material in fish feed due to their high content of fatty acids, particularly EPA and DHA $[60,61]$. The two mesopelagic fish species included in this paper presented the highest nutrient content of all sampled species for EPA and DHA, but also for vitamin $\mathrm{A}_{1}$, calcium, iodine, and iron, as in accordance with results from Alvheim et al. [62]. The high micronutrient content in these species may also be naturally attributed to the inclusion of various fish parts in the analyses, such as bones, skin, and viscera [63]. The eyes and liver of fish have previously been identified as particularly rich sources of vitamin A [4,64], with approximately $90 \%$ of the vitamin A located in the eyes and viscera of the small indigenous species, mola (Amblypharyngodon mola) [65]. The scientific literature on the biochemical composition of mesopelagic species and the distribution of mesopelagic diversity is particularly scarce [58]. From a food and nutrition security perspective, these types of studies are crucial to better understand the potential contribution such species may have on local food and nutrition security as a potentially harvestable resource for future commercial exploitation, either directly through human consumption or in a more indirect way as feed ingredients in aquaculture.

\subsection{Demersal Fish Species}

Bombay duck supplies the second largest marine fish catch in Bangladesh, representing over 12\% of total marine catch, and is considered a very affordable and easily available food, highly consumed by the poor $[22,66]$. In this paper, Bombay duck has the lowest nutrient concentrations of all the sampled species for protein, fat, all fatty acids, all vitamins, and all minerals. Other studies have reported a moisture content of approximately $87 \%$ for this species [43,67], which is considered very high [68], and in line with the mean dry matter of only $8.8 \%$ for the two samples of Bombay duck in this paper. This may explain the low nutritional quality in raw samples, as an inverse relationship between the 
water content and the content of fatty acids and protein exists; when the water content increases, the content of other nutrients decreases [69,70]. However, Bombay duck is commonly consumed both as fresh fish and as dried fish [22], and the chosen style may influence the nutrient yield immensely. Mohanty et al. presented a crude protein and fat content of $8.2 \%$ and $2.2 \%$, respectively, in wet samples of Bombay duck [67], similar to the values of $12.13 \%$ for protein and $2.15 \%$ for fat, as reported by Zaman et al. for wet samples [43]. When analysing the proximate nutrient content of dried Bombay duck sampled from the markets in Bangladesh, Siddique et al. (2012) reported a protein content of $57.03 \%$ and a fat content of $7.48 \%$, leading to the conclusion that due to increased nutrient-density per $100 \mathrm{~g}$ in dried Bombay duck compared to fresh Bombay duck, the consumption of dried Bombay duck is preferred [71].

\subsection{Potential Contribution to Recommended Nutrient Intakes}

The availability and thus the consumption of fish is dependent on socioeconomic status and prices, and fish is considered expensive compared to staple foods such as rice or maize $[72,73]$. Lower priced fish, such as small pelagic species and small indigenous species, both commonly consumed whole, are therefore preferentially purchased among poorer Bangladeshi households, thus playing an important role for food and nutrition security by diversifying the diets of the poor $[45,74,75]$. In Bangladesh, farmed fish is now cheaper and more readily available than marine fish due to expansions of aquaculture achieved over the last thirty years [73]. However, from a food and nutrition security perspective, it is important to consider not only the quantity of fish available in the country, but also how these fish are contributing to the nutritional needs of the population. Aquaculture systems produce only a limited variety of species, thus creating a shift away from the consumption of a diversity of species with a wide array of nutritional profiles that follows the diverse capture fisheries. Despite the rapid growth of aquaculture and increased overall consumption of fish in recent years, the overall lower nutritional quality of farmed fish compared to marine fish have likely exacerbated existing micronutrient deficiencies in Bangladesh [10,42]. With the exception of Bombay duck, we found that all marine species sampled may potentially contribute $\geq 100 \%$ to the RNI of vitamin $B_{12}$, EPA and DHA, and selenium, in addition to being able to contribute $\geq 50 \%$ to the RNIs of three or more nutrients simultaneously. All sampled species, aside from the demersal species, were also identified to potentially contribute $\geq 25 \%$ to the RNIs of six or more nutrients. This illustrates the wide diversity of nutrients that are available in marine fish and strengthens the evidence that marine fish provide high quantities of multiple critical nutrients required to improve micronutrient deficiencies. Further, fish enhances the bioavailability of minerals like iron and zinc from diets centred on starchy staples, thus including even small amounts of fish in the diet may enhance overall micronutrient bioavailability in the diet $[74,76]$. Due to the variability in micronutrient content with species, the nutritional quality needs to be examined for more marine fish species in order to optimise the complementary role fish may play in improving food and nutrition security in Bangladesh.

\subsection{Strengths and Limitations}

Representable sampling and proficient nutrient analyses are fundamental to yield high quality FCD that may be incorporated into local FCT. In this paper, the analytical value of each nutrient is based on three pooled samples consisting of a total of between 15 and 840 individual fish depending on the biomass of the species. This number is well above the standard value of ten units that is generally viewed as an appropriate number of samples used for FCD. Additionally, the species fringescale sardinella, slender rainbow sardine, and Bombay duck were sampled from multiple locations. This provides more data for each species' mean value, thus strengthening the confidence in the values [14]. However, pooled samples do not illustrate the individual variation within the species [77], and the nutrient content may also vary between different seasons, areas, maturity stages, and the sex of the fish $[68,70]$. All data presented in this paper were analysed at a national reference laboratory using accredited 
methods, thus representing important contributions to the Bangladeshi FCT and providing insight into the scarcely investigated nutrient composition of mesopelagic fish species.

\section{Conclusions}

The nutrient composition of several macro and micronutrients in seven marine fish species from Bangladesh, which are not included in the FCT for Bangladesh, are described in this paper. Among these seven species, five are commonly consumed by the Bangladeshi people and two are mesopelagic species not yet evaluated for their potential contribution to local food and nutrition security. The two mesopelagic species had the highest content of all fatty acids including EPA and DHA, vitamin A, calcium, iron, iodine, and selenium, whereas the demersal species had the lowest content of total fat and crude protein, all fatty acids, and all vitamins and minerals of the included species All species, with the exception of the demersal species Bombay duck ( $9 \%$ dry matter), were identified to potentially contribute substantially to the RNI of women of reproductive age for multiple micronutrients simultaneously if included in the diet. Our findings also suggest that the diversity of micronutrients in marine fish varies widely by species. It is important to recognise that our results are reported for raw fish, and both the processing and preparation of meals may impact the nutrient content of the species. More studies on the nutritional composition of fish following the value chain from fresh to prepared meals are needed to better understand the potential contribution of commonly consumed marine fish species and new potential marine resources to contribute to local food and nutrition security in Bangladesh.

Author Contributions: Conceptualization, M.K. and I.A.; methodology, M.K., S.H.T., and I.A.; validation, A.B. and M.K.; formal analysis, A.N. and A.M.R.; investigation, A.N. and A.A.M.R.; resources, A.B. and M.K.; data curation, A.N. and A.M.R.; writing-original draft preparation, A.A.M.R. and A.M.R.; writing-review and editing, A.N., A.A.M.R., I.A., A.M.R., L.M.P., A.B., A.M., S.H.T., T.H., T.S., and M.K.; visualization, A.N. and A.M.R.; supervision, M.K. and I.A.; project administration, M.K.; funding acquisition, M.K. All authors have read and agreed to the published version of the manuscript.

Funding: This research was funded by the Norwegian Agency for Development Cooperation (Norad).

Acknowledgments: The authors are grateful to Anne-Karin Syversen, Leikny Fjellstad, and Edel Erdal for their positive and enthusiastic engagement in organising the project samples stored and analysed at the IMR. We would also like to express our gratitude to all the laboratory technicians at IMR that have contributed to producing high-quality analytical data. The authors are also grateful to Magne Olsen for his help in creating the map of Bangladesh displaying the various sampling locations. Finally, we would like to acknowledge Gabriella Bianchi and Merete Tandstad for their help and guidance in the EAF-Nansen project.

Conflicts of Interest: The authors declare no conflict of interest. The funders had no role in the design of the study; in the collection, analyses, or interpretation of data; in the writing of the manuscript, or in the decision to publish the results.

\section{References}

1. Lund, E.K. Health benefits of seafood; is it just the fatty acids? Food Chem. 2013, 140, 413-420. [CrossRef] [PubMed]

2. Khalili Tilami, S.; Sampels, S. Nutritional Value of Fish: Lipids, Proteins, Vitamins, and Minerals. Rev. Fish Sci. Aquac. 2018, 26, 243-253. [CrossRef]

3. FAO; WHO. Report of the Joint FAO/WHO Expert Consultation on the Risks and Benefits of Fish Consumption; Food and Agriculture Organization of the United Nations: Rome, Italy; World Health Organization: Geneva, Switzerland, 2011.

4. Roos, N.; Islam, M.M.; Thilsted, S.H. Small indigenous fish species in Bangladesh: Contribution to vitamin A, calcium and iron intakes. J. Nutr. 2003, 133, 4021-4026. [CrossRef] [PubMed]

5. Fiedler, J.L.; Lividini, K.; Drummond, E.; Thilsted, S.H. Strengthening the contribution of aquaculture to food and nutrition security: The potential of a vitamin A-rich, small fish in Bangladesh. Aquaculture 2016, 452, 291-303. [CrossRef]

6. Belton, B.; van Asseldonk, I.J.M.; Thilsted, S.H. Faltering fisheries and ascendant aquaculture: Implications for food and nutrition security in Bangladesh. Food Policy 2014, 44, 77-87. [CrossRef] 
7. WorldFish. Bangladesh. Available online: https://www.worldfishcenter.org/country-pages/bangladesh (accessed on 7 October 2019).

8. Departement of Fisheries Bangladesh. Yearbook of Fisheries Statistics of Bangladesh 2016-2017; Fisheries Resources Survey System (FRSS), Department of Fisheries Bangladesh, Director General, DoF: Dhaka, Bangladesh, 2017; Volume 34, p. 129.

9. ICDDRB; UNICEF; GAIN. The Institute of Public Health and Nutrition; National Micronutrients Status Survey 2011-12; ICDDRB; UNICEF; GAIN: Dhaka, Bangladesh, 2013.

10. Bogard, J.R.; Farook, S.; Marks, G.C.; Waid, J.; Belton, B.; Ali, M.; Toufique, K.; Mamun, A.; Thilsted, S.H. Higher fish but lower micronutrient intakes: Temporal changes in fish consumption from capture fisheries and aquaculture in Bangladesh. PLoS ONE 2017, 12, e0175098. [CrossRef]

11. NIPORT; Mitra; Associates; ICF International. Bangladesh Demographic and Health Survey 2014; NIPORT; Mitra; Associated; ICF International: Dhaka, Bangladesh; Rockville, MD, USA, 2016.

12. WHO; UNICEF. Report of the Fourth Meeting of the WHO-UNICEF Technical Expert Advisory Group on Nutrition Monitoring (TEAM); WHO: Geneva, Switzerland; UNICEF: New York, NY, USA, 2017.

13. Magnani, R.; Oot, L.; Sethuraman, K.; Kabir, G.; Rahman, S. USAID Office for Peace; Food Security Country Framework for Bangladesh Fy 2015-2019; FCHI 360/FANTA: Washington, DC, USA, 2015.

14. Greenfield, H.; Southgate, D.A.T. Food Composition Data-Production, Management and Use, 2nd ed.; FAO: Rome, Italy, 2003.

15. INFOODS. Food Composition Challenges. Available online: http://www.fao.org/infoods/infoods/foodcomposition-challenges/en/ (accessed on 7 April 2019).

16. Murshed-E-Jahan, K.; Belton, B.; Viswanathan, K.K. Communication strategies for managing coastal fisheries conflicts in Bangladesh. Ocean Coast. Manag. 2014, 92, 65-73. [CrossRef]

17. Shaheen, N.; Rahim, A.; Mohiduzzaman, M.; Banu, C.; Bari, L.; Tukun, A.; Mannan, M.; Bhattacharjee, L.; Stadlmayr, B. Food Composition Table for Bangladesh. Available online: http://www.fao.org/fileadmin/ templates/food_composition/documents/FCT_10_2_14_final_version.pdf (accessed on 9 October 2018).

18. Shamsuzzaman, M.M.; Islam, M.M.; Tania, N.J.; Abdullah Al-Mamun, M.; Barman, P.P.; Xu, X. Fisheries resources of Bangladesh: Present status and future direction. Aquac. Fish. 2017, 2, 145-156. [CrossRef]

19. Roos, N.; Wahab, M.; Hossain, M.; Thilsted, S. Linking Human Nutrition and Fisheries: Incorporating Micronutrient-Dense, Small Indigenous Fish Species in Carp Polyculture Production in Bangladesh. Food Nutr. Bull. 2007, 28, 280-293. [CrossRef]

20. Thilsted, S.H. Improved management, increased culture and consumption of small fish species can improve diets of the rural poor. In Sustainable Diets and Biodiversity: Directions and Solutions for Policy, Research and Action; FAO; Biodiversity International: Rome, Italy, 2012; pp. 176-181.

21. Needham, S.; Funge-Smith, S.J. The Consumption of Fish and Fish Products in the Asia-Pacific Region Based on Household Surveys; RAP Publication 2015/12; FAO Regional Office for Asia and the Pacific: Bangkok, Thailand, $2014 ;$ p. 87.

22. Barange, M.; Fernandes, J.A.; Kay, S.; Hossain, M.A.R.; Ahmed, M.; Lauria, V. Marine Ecosystems and Fisheries: Trends and Prospects. In Ecosystem Services for Well-Being in Deltas: Integrated Assessment for Policy Analysis; Nicholls, R.J., Hutton, C.W., Adger, W.N., Hanson, S.E., Rahman, M.M., Salehin, M., Eds.; Springer International Publishing: Cham, Switzerland, 2018; pp. 469-488.

23. IMR. Mesopelagic Initiative: Unleashing New Marine Resources for a Growing Human Population. Available online: https://www.hi.no/filarkiv/2017/rad-bestander_og_ressurser-mesopelagic_initiative-unleashing new_marine_resources_for_a_growing_human_population.pdf/nb-no (accessed on 9 October 2019).

24. 24. Salvanes, A.G.V.; Kristoffersen, J.B. Mesopelagic Fishes, in Encyclopedia of Ocean Sciences; Steele, J.H., Thorpe, S.A., Turekian, K.K., Eds.; Academic Press Ltd.: San Diego, CA, USA, 2001.

25. Catul, V.; Gauns, M.; Karuppasamy, P.K. A review on mesopelagic fishes belonging to family Myctophidae. Rev. Fish Biol. Fish. 2011, 21, 339-354. [CrossRef]

26. Fischer, W.; Bianchi, G. (Eds.) FAO Species Identification Sheets for Fishery Purposes. In Western Indian Ocean (Fishing Area 51); Prepared and Printed with the Support of the Danish International Development Agency (DANIDA); FAO: Rome, Italy, 1984; Available online: http://www.fao.org/docrep/009/ad468e/ad468e00.htm (accessed on 3 April 2019).

27. Smith, J.L.B.; Smith, M.M.; Heemstra, P.C. Smiths' Sea Fishes; Penguin Random House: Cape Town, South Africa, 2003. 
28. Fricke, R.; Eschmeyer, W.; Van der Laan, R. Catalog of Fishes: Genera, Species, References; California Academy of Science: San Francisco, CA, USA, 2018.

29. Reksten, A.M.; Bøkevoll, A.; Frantzen, S.; Lundebye, A.-K.; Kögel, T.; Kolås, K.; Aakre, I.; Kjellevold, M. Sampling protocol for determination of nutrients and contaminants in fish and other seafood-The EAF Nansen Programme. unpublished, under review.

30. Norwegian Standard 9402 (Norsk Standard). Atlantic Salmon. Measurement of Fat and Colour, 1st ed.; Atlantisk Laks. Måling av Fett og Farge; Første Utgave: Bergen, Norway, 1994.

31. AOAC. Official Methods of Analysis. Crude Protein in Meat and Meat Products, Combustion Method, 16th ed.; Method 992.15; AOAC: Arlington, VA, USA, 1995.

32. Folch, J.; Lees, M.; Sloane Stanley, G. A simple method for the isolation and purification of total lipides from animal tissues. J. Biol. Chem. 1957, 226, 497-509.

33. Lie, Ø.; Lambertsen, G. Fatty acid composition of glycerophospholipids in seven tissues of cod (Gadus morhua), determined by combined high-performance liquid chromatography and gas chromatography. J. Chromatogr. B 1991, 565, 119-129. [CrossRef]

34. Torstensen, B.E.; Frøyland, L.; Ørnsrud, R.; Lie, Ø. Tailoring of a cardioprotective muscle fatty acid composition of Atlantic salmon (Salmo salar) fed vegetable oils. Food Chem. 2004, 87, 567-580. [CrossRef]

35. Comitè Europèen de Normalisation. Foodstuffs-Determination of Vitamin A by High Preformance Liquid Chromatography-Part 1: Measurement of All-Trans-Retinol and 13-Cis-Retinol; NS-EN 12823-1; Comitè Europèen de Normalisation: Brussels, Belgium, 2000.

36. Comitè Europèen de Normalisation. Foodstuffs-Determination of vitamin $D$ by high performance liquid chromatography-Measurement of cholecalciferol (D3) or ergocalciferol (D2); NS-EN 12821; Comitè Europèen de Normalisation: Brussels, Belgium, 2009.

37. AOAC. Methods for the Microbiological Analyses of Selected Nutrients; AOAC: Arlington, VA, USA, 1996; pp. 63-65.

38. Julshamn, K.; Maage, A.; Norli, H.S.; Grobecker, K.H.; Jorhem, L.; Fecher, P. Determination of arsenic, cadmium, mercury, and lead by inductively coupled plasma/mass spectrometry in foods after pressure digestion: NMKL interlaboratory study. J. AOAC Int. 2007, 90, 844-856. [CrossRef]

39. Julshamn, K.; Dahl, L.; Eckhoff, K. Determination of iodine in seafood by inductively coupled plasma/mass spectrometry. J. AOAC Int. 2001, 84, 1976-1983. [CrossRef]

40. WHO; FAO. Vitamin and Mineral Requirements in Human Nutrition, 2nd ed.; WHO: Geneva, Switzerland; FAO: Rome, Italy, 2004; Available online: http://www.fao.org/ag/humannutrition/3665904427f866c8b2539d8e47d408cad5f3f9.pdf (accessed on 20 March 2019).

41. EFSA. Scientific Opinion on Dietary Reference Values for fats, including saturated fatty acids, polyunsaturated fatty acids, monounsaturated fatty acids, trans fatty acids, and cholesterol. EFSA J. 2010, 8, 1461.

42. Bogard, J.; Thilsted, S.H.; Marks, G.C.; Wahab, M.A.; Hossain, M.A.R.; Jakobsen, J.; Stangoulis, J. Nutrient composition of important fish species in Bangladesh and potential contribution to recommended nutrient intakes. J. Food Comp. Anal. 2015, 42, 120-133. [CrossRef]

43. Zaman, M.; Naser, N.; Abdullah, A.T.; Khan, N. Nutrient contents of some popular freshwater and marine fish species of Bangladesh. J. Zool. 2014, 42, 251-259. [CrossRef]

44. Anwarul, G.M.M.; Hossain, M.; Farha, J.; Mohajira, B. Nutritional analysis of three different cultured fishes of Bangladesh. Int. Adv. Res. J. Sci. Eng. Technol. 2015, 2, 1-4.

45. Roos, N.; Islam, M.; Thilsted, S.H. Small fish is an important dietary source of vitamin A and calcium in rural Bangladesh. Int. J. Food Sci. Nutr. 2003, 54, 329-339. [CrossRef] [PubMed]

46. Ahmed, S.; Rahman, A.F.M.; Mustafa, M.; Hossain, M.B.; Nahar, N. Nutrient Composition of Indigenous and Exotic Fishes of Rainfed Waterlogged Paddy Fields in Lakshmipur, Bangladesh. WJZ 2012, 7, 135-140.

47. Alam, S.M.D.; Karim, M.H.; Chakrabortty, A.; Amin, R.; Hasan, S. Nutritional Characterization of the Long-whiskered Catfish Sperata aor: A Commercially Important Freshwater Fish of Bangladesh. Int. J. Food Sci. Nutr. 2016, 6, 1-8.

48. Kamal, D.; Khan, A.N.; Rahman, M.A.; Ahamed, F. Biochemical Composition of Some Small Indigenous Fresh Water Fishes from the River Mouri, Khulna, Bangladesh. PJBS 2007, 10, 1559-1561.

49. Mazumder, M.S.A.; Rahmani, M.M.; Ahmed, A.T.A.; Begum, M.; Hossain, M.A. Proximate composition of some small indigenous fish species in Bangladesh. Int. J. Sustain. Crop Prod. 2008, 3, 18-23. 
50. Malaysian Food Composition Database. Food Details Nutrient Compositions-Megalaspis Cordyla. Available online: http://myfcd.moh.gov.my/index.php/1997-food-compositon-database/480-scad,-hairtail(cincaru)-;-megalaspis-cordyla (accessed on 2 April 2019).

51. National Technical Working Group of Malaysian Food Composition Database. Protocol for Sampling and Methods of Analysis for Malaysian Food Composition Database. Available online: https:/www.imr.gov.my/ images/uploads/Protocol_Sampling_MY_FCD.pdf (accessed on 4 May 2019).

52. La Frano, M.R.; Cai, Y.; Burri, B.J.; Thilsted, S.H. Discovery and biological relevance of 3,4-didehydroretinol (vitamin A2) in small indigenous fish species and its potential as a dietary source for addressing vitamin A deficiency. Int. J. Food Sci. Nutr. 2018, 69, 253-261. [CrossRef]

53. Larsen, T.; Thilsted, S.H.; Kongsbak, K.; Hansen, M. Whole small fish as a rich calcium source. Br. J. Nutr. 2000, 83, 191-196. [CrossRef]

54. Martínez-Valverde, I.; Jesús Periago, M.; Santaella, M.; Ros, G. The content and nutritional significance of minerals on fish flesh in the presence and absence of bone. Food Chem. 2000, 71, 503-509. [CrossRef]

55. Malde, M.K.; Bügel, S.; Kristensen, M.; Malde, K.; Graff, I.E.; Pedersen, J.I. Calcium from salmon and cod bone is well absorbed in young healthy men: A double-blinded randomised crossover design. Nutr. Metab. 2010, 7, 61. [CrossRef]

56. Palani Kumar, M.; Ruba Annathai, A.; Jeya Shakila, R.; Shanmugam, S. Proximate and Major Mineral Composition of 23 Medium Sized Marine Fin Fishes Landed in the Thoothukudi Coast of India. J. Nutr. Food Sci. 2014, 4, 1-8.

57. Hansen, M.; Thilsted, S.H.; Sandstrom, B.; Kongsbak, K.; Larsen, T.; Jensen, M.; Sørensen, S.S. Calcium absorption from small soft-boned fish. J. Trace Elem. Med. Biol. 1998, 12, 148-154. [CrossRef]

58. St. John, M.A.; Borja, A.; Chust, G.; Heath, M.; Grigorov, I.; Mariani, P.; Martin, A.P.; Santos, R.S. A Dark Hole in Our Understanding of Marine Ecosystems and Their Services: Perspectives from the Mesopelagic Community. Front. Mar. Sci. 2016, 3, 31.

59. El-Mowafi, A.; Nanton, D.; Berntssen, M. Evaluation of lantern fish (Benthosema Pterotum) as marine source in fish feeds: Nutrient composition and contaminants assessment. FAR 2010, 29, 12-23.

60. Lea, M.-A.; Nichols, P.D.; Wilson, G. Fatty acid composition of lipid-rich myctophids and mackerel icefish (Champsocephalus gunnari)—Southern Ocean food-web implications. Polar Biol. 2002, 25, 843-854. [CrossRef]

61. Koizumi, K.; Hiratsuka, S.; Saito, H. Lipid and fatty acids of three edible myctophids, Diaphus watasei, Diaphus suborbitalis, and Benthosema pterotum: High levels of icosapentaenoic and docosahexaenoic acids. J. Oleo Sci. 2014, 63, 461-470. [CrossRef]

62. Alvheim, A.R.; Kjellevold, M.; Strand, E.; Sanden, M.; Wiech, M. Mesopelagic Species and Their Potential Contribution to Food and Feed Security-A Case Study from Norway. Foods 2020, 9, 344. [CrossRef]

63. Reksten, A.M.; Somasanduram, T.; Kjellevold, M.; Nordhagen, A.; Bøkevoll, A.; Pincus, L.M.; Rizwan, A.A.M.; Mamun, A.; Thilsted, S.H.; Htut, T.; et al. Nutrient composition of 19 fish species from Sri Lanka and potential contribution to food and nutrition security. J. Food Compos. Anal. 2020, 91, 103508. [CrossRef]

64. Roos, N.; Wahab, M.A.; Chamnan, C.; Thilsted, S.H. The role of fish in food-based strategies to combat vitamin A and mineral deficiencies in developing countries. J. Nutr. 2007, 137, 1106-1109. [CrossRef]

65. Roos, N.; Leth, T.; Jakobsen, J.; Thilsted, S.H. High vitamin A content in some small indigenous fish species in Bangladesh: Perspectives for food-based strategies to reduce vitamin A deficiency. Int. J. Food Sci. Nutr. Eng. 2002, 53, 425-437. [CrossRef]

66. Fernandes, J.; Kay, S.; Hossain, M.; Ahmed, M.; Cheung, W.; Lazar, A.; Barange, M. Projecting marine fish production and catch potential in Bangladesh in the 21st century under long-term environmental change and management scenarios. ICES J. Mar. Sci. 2015, 73, 1357-1369. [CrossRef]

67. Mohanty, B.P.; Mahanty, A.; Ganguly, S.; Mitra, T.; Karunakaran, D.; Anandan, R. Nutritional composition of food fishes and their importance in providing food and nutritional security. Food Chem. 2019, 30, 561-570. [CrossRef] [PubMed]

68. Murray, J.; Burt, J.R. The Composition of Fish. Available online: http://www.fao.org/3/x5957e00.htm\#Contents (accessed on 7 May 2019).

69. Huss, H.H. Quality and Quality Changes in Fresh Fish: FAO Fisheries Technical Paper-348; FAO: Rome, Italy, 1995. Available online: http://www.fao.org/docrep/V7180E/V7180E00.HTM\#Contents (accessed on 29 October 2019). 
70. EFSA. Opinion of the Scientific Panel on contaminants in the food chain [CONTAM] related to the safety assessment of wild and farmed fish. EFSA J. 2005, 3. [CrossRef]

71. Siddique, M.A.M.; Mojumder, P.; Zamal, H. Proximate Composition of Three Commercially Available Marine Dry Fishes (Harpodon nehereus, Johnius dussumieri and Lepturacanthus savala). Am. J. Food Technol. 2012, 7, 429-436. [CrossRef]

72. Speedy, A.W. Global production and consumption of animal source foods. J. Nutr. 2003, 133, 4048-4053. [CrossRef]

73. Beveridge, M.C.M.; Thilsted, S.H.; Phillips, M.J.; Metian, M.; Troell, M.; Hall, S.J. Meeting the food and nutrition needs of the poor: The role of fish and the opportunities and challenges emerging from the rise of aquaculture. J. Fish Biol. 2013, 83, 1067-1084. [CrossRef]

74. Kawarazuka, N.; Bene, C. The potential role of small fish species in improving micronutrient deficiencies in developing countries: Building evidence. Public Health Nutr. 2011, 14, 1927-1938. [CrossRef]

75. Kabahenda, M.K.; Amega, R.; Okalany, E.; Husken, S.M.C.; Heck, S. Protein and Micronutrient Composition of Low-Value Fish Products Commonly Marketed in the Lake Victoria Region. World J. Agric. Sci. 2011, 7, 521-526.

76. Navas-Carretero, S.; Perez-Granados, A.M.; Sarria, B.; Carbajal, A.; Pedrosa, M.M.; Roe, M.A.; Fairweather-Tait, S.J.; Vaquero, M.P. Oily fish increases iron bioavailability of a phytate rich meal in young iron deficient women. J. Am. Coll. Nutr. 2008, 27, 96-101. [CrossRef]

77. Caudill, S.P. Use of pooled samples from the National Health and Nutrition Examination Survey. Stat. Med. 2012, 31, 3269-3277. [CrossRef]

(C) 2020 by the authors. Licensee MDPI, Basel, Switzerland. This article is an open access article distributed under the terms and conditions of the Creative Commons Attribution (CC BY) license (http://creativecommons.org/licenses/by/4.0/). 